\title{
Risk factors of medication-related osteonecrosis of the jaw: a retrospective study in a Turkish subpopulation
}

\author{
Onur Şahin ${ }^{1}$, Onur Odabaşı, Toghrul Aliyev ${ }^{1}$, Birkan Tatar ${ }^{1}$ \\ ${ }^{\prime}$ Department of Oral and Maxillofacial Surgery, Faculty of Dentistry, İzmir Katip Çelebi University, İzmir, \\ ${ }^{2}$ Department of Oral and Maxillofacial Surgery, Ankara Yldirm Beyazit University, Ankara, Turkey
}

\begin{abstract}
J Korean Assoc Oral Maxillofac Surg 2019;45:108-115)
Objectives: Medication-related osteonecrosis of the jaw (MRONJ) is a well-known side effect of certain drugs that are used to influence bone metabolism to treat osteometabolic disease or cancers. The purpose of our study was to investigate how high-concentration and low-concentration bisphosphonate (BP) intake affects the disease severity.

Materials and Methods: Data collected from the medical records of 52 patients treated with BPs, antiresorptive, antiangiogenic drugs and diagnosed with MRONJ were included in this study. Age, sex, type of systemic disease, type of drug, duration of drug treatment, jaw area with MRONJ, drug administration protocol, and MRONJ clinical and radiological findings were obtained. Patients were divided into two groups: anti-neoplastic (Group I, $\mathrm{n}=23$ ) and anti-osteoporotic (Group II, n=29). Statistical evaluations were performed using the IBM SPSS ver. 21.0 program.

Results: In both groups, more females had MRONJ. MRONJ was found in the mandibles of 30 patients (Group I, n=14; Group II, n=16). When we classified patients according to the American Association of Oral and Maxillofacial Surgeons staging system, significant differences were seen between groups $\left(\chi^{2}=12.23, P<0.01\right)$. More patients with advanced stage (stage 2-3) MRONJ were found in Group I $(60.9 \%)$.

Conclusion: According to our results, high-concentration BP intake, age and duration of drug intake increased disease severity.
\end{abstract}

Key words: Bisphosphonate-associated osteonecrosis of the jaw, Osteonecrosis, Risk factors, Zoledronic acid

[paper submitted 2018. 7. 2 / revised 2018. 7. 30 / accepted 2018. 7. 31]

\section{Introduction}

Bisphosphonates (BPs) are the most commonly used antiresorptive drugs for the prevention of skeletal complications in many diseases. The term bisphosphonate-related osteonecrosis of the jaw (BRONJ) was first coined by Marx ${ }^{1}$ in 2003. An increase in localized osteonecrosis in jaw bones is related to BPs. The adverse effects of bisphosphonates on quality of life and increased morbidity, the researchers have led to the investigation of early diagnosis and effective treat-

\footnotetext{
Onur Şahin

Department of Oral and Maxillofacial Surgery, Faculty of Dentistry, Izmir Katip Çelebi University, Aydınlıkevler Mahallesi, Cemil Meriç Bulvarı, 6780 Sokak, \#48, İmir 35640, Turkey

TEL: +90-(232)-325-25-35 FAX: +90-5054410192

E-mail:onursahin43@hotmail.com

ORCID: https://orcid.org/0000-0001-7816-1443

(c) This is an open-access article distributed under the terms of the Creative Commons Attribution Non-Commercial License (http://creativecommons.org/ licenses/by-nc/4.0/), which permits unrestricted non-commercial use, distribution, and reproduction in any medium, provided the original work is properly cited.

Copyright (C) 2019 The Korean Association of Oral and Maxillofacial Surgeons. All rights reserved.
}

ment strategies. In addition to BPs, other antiresorptive (e.g., denosumab) and antiangiogenic drugs (e.g., bevacizumab and sunitinib) can cause osteonecrosis, so the American Association of Oral and Maxillofacial Surgeons (AAOMS) proposed using medication-related osteonecrosis of the jaw (MRONJ) as the terminology to replace BRONJ ${ }^{2}$. Drugs that cause osteonecrosis of the jaw are defined as antiresorptive and antiangiogenic drugs. Drugs such as high-concentration and lowconcentration BPs and denosumab are antiresorptive drugs. Intravenous (IV) BPs are frequently used to treat hypercalcemia associated with malignant tumors due to their antitumoral effects in the treatment of cancers with bone metastases including breast, prostate and lung, and to prevent skeletal complications in multiple myeloma. Oral BPs are frequently associated with osteoporosis and osteopenia and rarely, with treatment of Paget's disease and osteogenesis imperfecta ${ }^{3}$. Osteonecrosis of the jaw is a rare condition that develops as a result of impaired blood supply to the mandible and maxilla. The incidence of osteonecrosis in individuals treated with BP therapy varies between $1 \%$ and $21 \%{ }^{4}$. Bisphosphonates and other antiresorptive drugs increase apoptosis by inhibiting 
osteoclast differentiation and function, and these events cause bone resorption and reduced bone remodeling. Although osteoclast differentiation and function play a crucial part in the remodeling of all the bones in the skeletal system, osteonecrosis is most commonly observed in jaw bones owing to high bone metabolism in jaw bones ${ }^{5}$.

In 2014, three criteria were defined by the AAOMS for differential diagnosis of MRONJ from other diseases that can cause clinical osteonecrosis. These criteria are: the presence of antiresorptive and antiangiogenic drug use in the patient's medical history, clinical exposure of the bone site for more than 8 weeks, and no radiotherapy or metastasis in the jaw bone $^{2}$. In 2006, a staging system and treatment protocol was developed by Ruggiero et al. ${ }^{6}$. This staging system was updated by the AAOMS in 2007, 2009, and 2014. Our study was based on the staging system updated in $2014^{2}$.(Table 1)

The purpose of our study was to investigate how highconcentration and low-concentration BP intake affected the severity of MRONJ. Patient records were evaluated retrospectively for demographic data, medical information, clinical and radiological examination findings, drug type, usage information, and related disease.

Table 1. American Association of Oral and Maxillofacial Surgeons staging system

\begin{tabular}{ll}
\hline At-risk category & Asymptomatic stage without necrotic bone \\
Stage 0 & No exposure bone, but clinical and radiological nonspecific findings and symptoms \\
Stage 1 & In asymptomatic patients with no signs of infection, presence of exposed and necrotic bone \\
Stage 2 & Symptomatic stage, radiological findings, exposed, necrotic bone, fistula and infection are localized in the alveolar bone region \\
Stage 3 & $\begin{array}{r}\text { The exposure may range from necrotic bone, fistula and infection, symptomatic stage, necrotic bone from the alveolar bone to } \\
\text { the lower border of the mandible, to the ramus or to the maxillary sinus, to the zygomatic bone. There may be pathological } \\
\text { fractures, extra-oral fistula, oral-antral or oral-nazal relationship and osteolytic area extending to the lower border of the } \\
\text { mandible or sinus base. }\end{array}$
\end{tabular}

Data from the article of Ruggiero et al. (J Oral Maxillofac Surg 2014;72:1938-56) .

Onur Şahin et al: Risk factors of medication-related osteonecrosis of the jaw: a retrospective study in a Turkish subpopulation. J Korean Assoc Oral Maxillofac Surg 2019
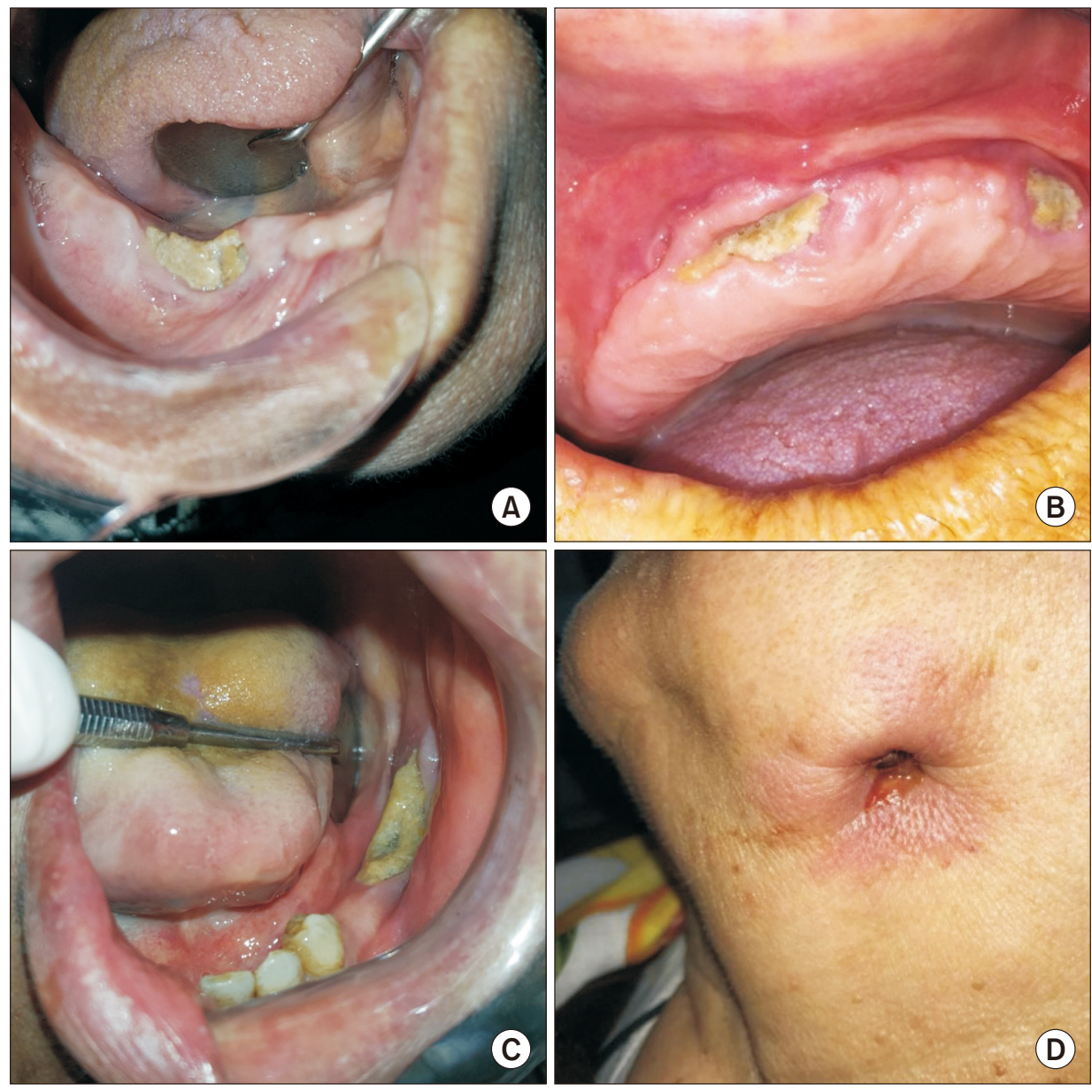

Fig. 1. Clinical findings of some medication-related osteonecrosis of the jaw (MRONJ) patients in this study. A. MRONJ lesion developed that following tooth extraction at right mandibular premolar area. B. Clinical image of maxillary exposed, necrotic bone in a MRONJ patient due to prosthesis trauma. C. Stage II MRONJ in a female patient treated with alendronate for osteoporosis. D. Extra-oral draining fistula at the submandibular region of the jaw in a patient with stage $3 \mathrm{MRONJ}$.

Onur Sahin et al: Risk factors of medication-related osteonecrosis of the jaw: a retrospective study in a Turkish subpopulation. J Korean Assoc Oral Maxillofac Surg 2019 


\section{Materials and Methods}

This retrospective study included 52 patients over a 5-year period (January 2013 to December 2017) using database records of the Department of Oral and Maxillofacial Surgery, Faculty of Dentistry, İzmir Katip Çelebi University (İzmir, Turkey). Patients who were diagnosed with MRONJ were included. The study was approved by the Ethical Committee of İzmir Katip Çelebi University (approval No. 207), and it was conducted in accordance with the Declaration of Helsinki.

Data collected from the medical records of patients included age, sex, type of systemic disease, type of drug, duration of drug treatment, jaw area seen with MRONJ, drug administration protocol, MRONJ staging and radiological findings. According to information collected on patients, we determined staging and treatment systems as defined by the AAOMS position paper, on MRONJ for each patient ${ }^{2}$.(Table 1) Patients with head and neck radiotherapy or necrotic bone areas less than 8 weeks were not included. Several studies have been published using the AAOMS staging system updated in 2014. However, as far as we know, few studied the use of the staging system to classify patients into antineoplastic (Group I, n=23) and anti-osteoporotic (Group II, $n=29$ ) groups. We analyzed sex, age, related disease, type of drug, location of MRONJ, local etiological factors and clinical and radiological findings and their relationships to investigate how high-concentration and low-concentration BP intake affected disease severity.(Fig. 1, 2, Table 2)

Statistical evaluations were performed with the IBM SPSS Statistics program (ver. 21.0; IBM Corp., Armonk, NY, USA). The Kolmogorov-Smirnov test was applied to all continuous variables to determine if distributions met the normality assumption. Parametric tests were used for variables that met normality assumptions. Comparisons of categorical variables were made with chi-square or Fisher's exact tests. The Student's t-test was used to compare quantitative variables between Group I and Group II. All data were evaluated at a significance level of $P<0.05$.

\section{Results}

Of the 52 patients included in the study, 7 were male and
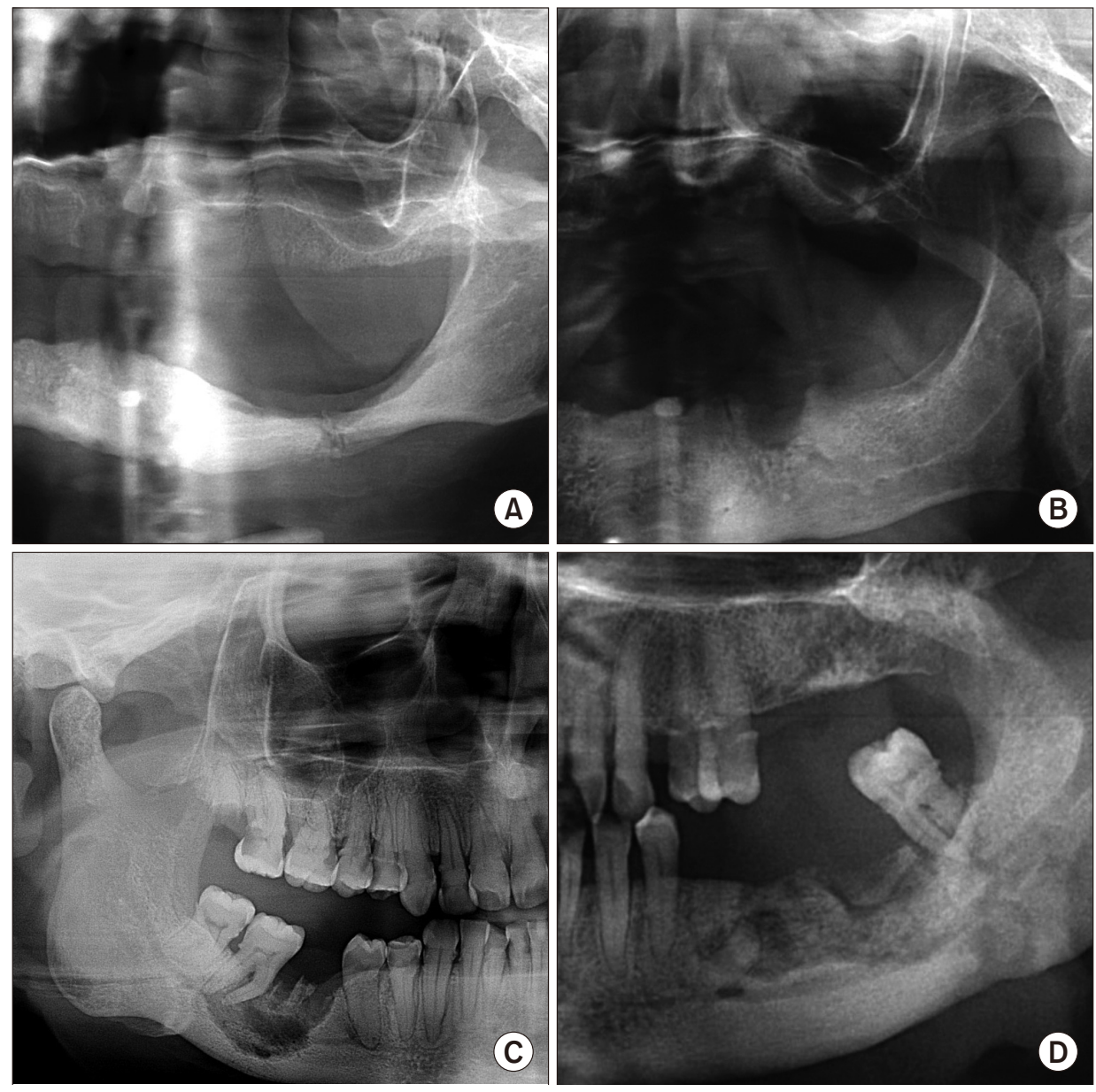

Fig. 2. Radiological findings of some medication-related osteonecrosis of the jaw (MRONJ) patients in this study. A. Panoramic radiograph demonstrating a pathologic fracture of the left molar region of the mandible in a patient with metastatic breast and stage $3 \mathrm{MRONJ}$. B. Panoramic radiography demonstrated osteolytic process at the left mandible. C. Panoramic radiography showing persisting alveolar socket in the mandible. D. Panoramic radiography demonstrated diffuse osteolysis of the jaw with areas of bone sclerosis. Onur Sahin et al: Risk factors of medication-related osteonecrosis of the jaw: a retrospective study in a Turkish subpopulation. J Korean Assoc Oral Maxillofac Surg 2019 
Table 2. Patient characteristics and outcomes

\begin{tabular}{|c|c|c|c|c|}
\hline & Group I (n=23) & Group II (n=29) & t or $\chi^{2}$ & $P$-value \\
\hline Age (yr) & $61.91 \pm 10.84$ & $62.72 \pm 10.55$ & $\mathrm{t}=-0.272$ & $>0.05$ \\
\hline \multicolumn{5}{|l|}{ Sex } \\
\hline Male & $5(21.7)$ & $2(6.9)$ & & $>0.05^{1}$ \\
\hline Female & $18(78.3)$ & $27(93.1)$ & & \\
\hline \multirow[t]{8}{*}{ Disease } & Lung cancer: 3 (13.0) & Osteoporosis: 29 (100) & $\chi^{2}=31.85$ & $<0.01$ \\
\hline & Renal cancer: 1 (4.3) & & & \\
\hline & Breast cancer: 6 (26.1) & & & \\
\hline & Multiple myeloma: 3 (13.0) & & & \\
\hline & Nasopharynx cancer: 1 (4.3) & & & \\
\hline & Osteoporosis: 6 (26.1) & & & \\
\hline & Over cancer: 1 (4.3) & & & \\
\hline & Prostate cancer: 2 (8.7) & & & \\
\hline \multirow[t]{3}{*}{ Drug } & Ibandronate: 12 (52.2) & Ibandronate: 5 (17.2) & $\chi^{2}=37.70$ & $<0.01$ \\
\hline & Zoledronate: 9 (39.1) & Alendronate: 21 (72.4) & & \\
\hline & Zoledronate+denosumab: 2 (8.7) & Residronate: 3 (10.3) & & \\
\hline \multicolumn{3}{|l|}{ Location of MRONJ } & $\chi^{2}=3.79$ & $>0.05$ \\
\hline Mandible & $14(60.9)$ & $16(55.2)$ & & \\
\hline Upper jaw & $8(34.8)$ & $12(41.4)$ & & \\
\hline Both & $1(4.3)$ & $1(3.4)$ & & \\
\hline \multicolumn{3}{|l|}{ Local etiologic factor } & $\chi^{2}=1.64$ & $>0.05$ \\
\hline Dental extractions & $14(60.9)$ & $15(51.7)$ & & \\
\hline Prosthesis & $6(26.1)$ & $6(20.7)$ & & \\
\hline Spontaneous & $2(8.7)$ & $8(27.6)$ & & \\
\hline Implant treatment & $1(4.3)$ & $0(0)$ & & \\
\hline \multicolumn{5}{|c|}{ Clinical and radiographic sign } \\
\hline Pain & $15(65.2)$ & $17(58.6)$ & $\chi^{2}=0.24$ & $>0.05$ \\
\hline Infection & $4(17.4)$ & $1(3.4)$ & $\chi^{2}=2.87$ & $>0.05$ \\
\hline Suppuration & $8(34.8)$ & $2(6.9)$ & & $<0.05^{1}$ \\
\hline Fracture & $2(8.7)$ & $0(0)$ & & $>0.05^{1}$ \\
\hline Exposed bone & $18(78.3)$ & $18(62.1)$ & $\chi^{2}=1.58$ & $>0.05$ \\
\hline
\end{tabular}

(Group I: anti-neoplastic, Group II: anti-osteoporotic, MRONJ: medication-related osteonecrosis of the jaw)

${ }^{1}$ Fisher's exact test.

Values are presented as mean \pm standard deviation or number $(\%)$.

Onur Sahin et al: Risk factors of medication-related osteonecrosis of the jaw: a retrospective study in a Turkish subpopulation. J Korean Assoc Oral Maxillofac Surg 2019

45 were female. Of these, 29 were given BP or antiresorptive drugs orally ( 2 males and 27 females) and 23 were given drugs by IV ( 5 males and 18 females). The mean age of patients was $61.91 \pm 10.84$ years (range, $44-80$ years) in Group I and 62.72 \pm 10.55 years (range, 40-76 years) in Group II. In Group I, osteonecrosis was observed in the mandible in 14 patients, in the maxilla in 8 patients and in both jaws in one patient. In Group II, 16 patients had mandibular and 12 patients had maxillary MRONJ, and one patient had MRONJ findings in both jaws. MRONJ was found in the mandibles in 30 patients. No significant difference was seen between the groups by location $(P>0.05)$.

All patients in Group II were taking medications to prevent osteoporosis. Patients in Group I were taking high-concentration BPs for oncological reasons. Of the patients, 3 were treated for lung cancer, 1 for renal cancer, 6 for breast cancer, 3 for multiple myeloma, 1 for nasopharynx cancer, 6 for osteoporosis, 1 for ovarian cancer, and 2 for prostate cancer.

All patients in Group II were treated for osteoporosis. In Group I, 6 patients were taking parenteral medication for osteoporosis, 3 using ibandronate and 3 zoledronate. Patients in Group I were treated 2 to 10 years (mean, 29 months) and patients in Group II were treated 1 to 8 years (mean, 44 months).

Of patients in Group I, 12 were using ibandronate (52.2\%), 9 zoledronate (39.1\%), and 2 a combination of zoledronate and denosumab (8.7\%). Of the patients in Group II, 5 were using ibandronate (17.2\%), 21 alendronate (72.4\%), and 3 residronate $(10.3 \%)$.

When local etiologic factors causing MRONJ were examined, 14 patients in Group I showed MRONJ after tooth extraction, 6 after receiving dental prosthesis, 2 spontaneously and 1 after dental implant treatment. Of patients in Group II, 15 had MRONJ post tooth extraction, 6 after receiving prosthetics, and 8 had spontaneous osteonecrosis. In both groups, the etiologic factor that caused MRONJ was mainly tooth extraction. The difference between the groups was not significant.

When clinical and radiological findings were examined for pain, infection, suppuration, fracture and exposed bone, pain 
Table 3. Staging of patients with high-concentration (Group I) and low-concentration (Group II) bisphosphonate use

\begin{tabular}{cccc}
\hline Stage & Group I $(\mathrm{n}=23)$ & Group II $(\mathrm{n}=29)$ & Total $(\mathrm{n}=52)$ \\
\hline 0 & $3(13.0)$ & $11(37.9)$ & $14(26.9)$ \\
1 & $6(26.1)$ & $13(44.8)$ & $19(36.5)$ \\
2 & $10(43.5)$ & $5(17.2)$ & $15(28.8)$ \\
3 & $4(17.4)$ & $0(0)$ & $4(7.7)$ \\
\hline
\end{tabular}

$\chi^{2}=12.23, P<0.01$.

Onur Şahin et al: Risk factors of medication-related osteonecrosis of the jaw: a retrospective study in a Turkish subpopulation.J Korean Assoc Oral Maxillofac Surg 2019

and exposed bone were observed in both groups. No significant difference was found between the groups except for suppuration $(P>0.05)$. (Table 2)

When we classified patients according to the AAOMS staging system, significant differences were seen between the groups $\left(\chi^{2}=12.23, P<0.01\right)$. Advanced stage (stage 2-3) disease was found at higher rates in Group I (60.9\%). Early stage cases were significantly higher in Group II (82.7\%). (Table 3)

A significant difference was found in the relationship between age and stage. The incidence of stage 2 and 3 disease increased with age in patients with MRONJ $\left(\chi^{2}=17.73\right.$, $P<0.05$ ).(Table 4 ) We found that 3 patients smoked a package of 20 cigarettes per day for more than 10 years: 2 had stage 2 and 1 patient had stage 3 disease.

\section{Discussion}

Although the first case of MRONJ was reported in 2003, MRONJ physiopathology is not fully understood, and potential mechanisms are still being discussed ${ }^{1}$. The physiopathology of MRONJ is explained as modified bone remodeling or suppression of bone resorption, inflammation or infection, inhibition of angiogenesis, soft tissue toxicity, congenital or acquired immunodeficiency, microtrauma, and vitamin D insufficiency ${ }^{7}$.

According to the AAOMS classification, risk factors for osteonecrosis formation in jaw bones due to use of BPs are medication-related, local, and demographic/systemic ${ }^{8}$. Risk factors associated with drug use include type of BP used, method of drug administration, and duration of treatment. In particularly, intravenous use of BPs increases risk ${ }^{9,10}$. Local risk factors include periodontal diseases, inflammatory dental diseases (such as dental abscesses), torus mandibularis and palatinus, dental caries, dental alveolar surgery (tooth extraction, dental implant application, periodontal surgery including bone tissue, periapical surgery), and traumatic factors such as
Table 4. Relationship between disease stage and age groups

\begin{tabular}{lccccc}
\hline \multirow{2}{*}{ Stage } & \multicolumn{5}{c}{ Age group $(\mathrm{yr})$} \\
\cline { 2 - 5 } & $40-50$ & $51-60$ & $61-70$ & $71-80$ & \\
\hline 0 & 3 & 9 & 2 & 0 & 14 \\
1 & 2 & 3 & 11 & 3 & 19 \\
2 & 1 & 5 & 5 & 4 & 15 \\
3 & 0 & 1 & 1 & 2 & 4 \\
Total & 6 & 18 & 19 & 9 & 52 \\
\hline
\end{tabular}

$\chi^{2}=17.73, P<0.05$.

Onur Şahin et al: Risk factors of medication-related osteonecrosis of the jaw: a retrospective study in a Turkish subpopulation. J Korean Assoc Oral Maxillofac Surg 2019

bone exostoses, mylohyoid protrusion, or unadapted dental prostheses ${ }^{11}$.

Demographic and systemic risk factors are defined as diabetes mellitus, immunosuppression, anemia and thalassemia, malnutrition, osteoporosis/osteopenia diagnosed with cancer, sex, chronic corticosteroid use, radiotherapy, estrogen therapy, smoking, cancer diagnosis, coagulation disorders, blood anomalies, vascular and connective tissue diseases, and hypothyroidism ${ }^{12}$. This study also investigated how these risk factors affect patient low-concentration and high-concentration BP intake.

Osteoporosis can occur in individuals over 50 years of age and antiresorptive agents are often preferred for treatment. In patients with osteoporosis using low-concentration BPs, the incidence of MRONJ is between $0.00038 \%$ and $0.1 \%$. For use longer than 4 years, the incidence is reported to be $0.21 \%{ }^{13}$.

In patients with osteoporosis using high-concentration BPs, MRONJ incidence is reported to be $0.017 \%$ in a 3 -year follow-up period. This ratio remained unchanged in a 6-year follow-up ${ }^{14}$. In our study, 23 patients with MRONJ were using high-concentration and 29 patients were using lowconcentration BPs. In Group I, the duration of use of BPs or antiresorptive drugs was 29 months. In Group II, duration of use was 44 months, similar to Zervas et al. ${ }^{12}$ (24 months). Our results are not consistent with those of Berenson et al. ${ }^{15}$ (18 months of zoledronate) and Dimopoulos et al. ${ }^{16}$ (53.4 months of pamidronate and zoledronate therapy). The high MRONJ incidence for patients taking low-concentration BPs could be linked to a longer duration of drug use and greater influence of local etiological factors. Also, we examined patients who were diagnosed with MRONJ. When we classified patients according to the AAOMS staging system, Group I had a higher rate of advanced disease stage than Group II. We found 14 patients $(60.9 \%)$ with advanced-stage (2 or 3) disease in Group I and 5 patients (17.2\%) in Group II. 


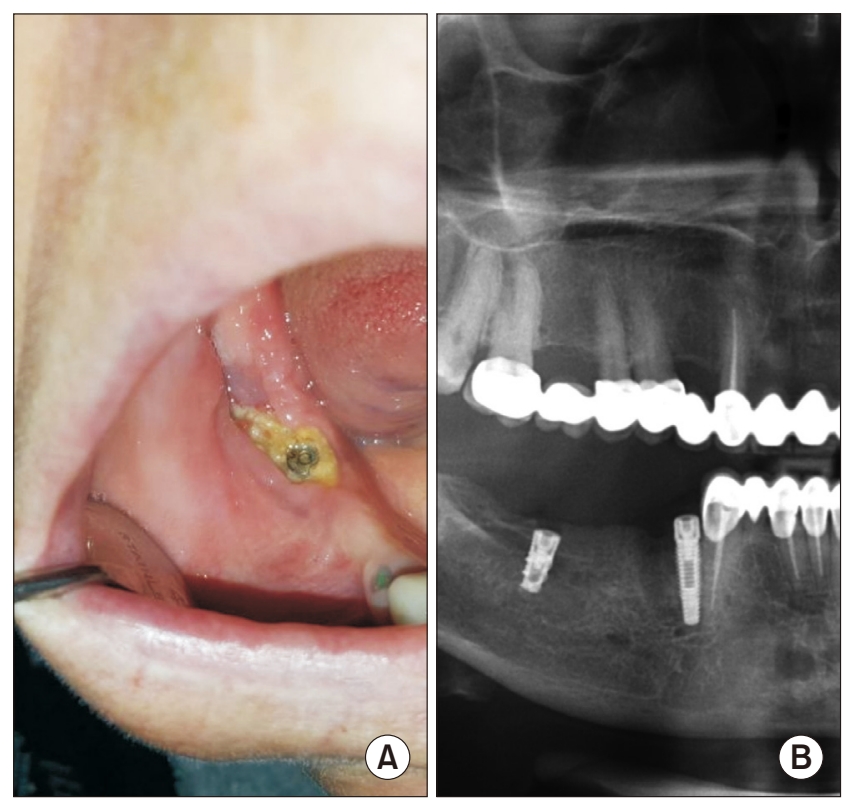

Fig. 3. Clinical and radiologic findings after dental implant treatment in medication-related osteonecrosis of the jaw (MRONJ) patients using zoledronate and denosumab. A. Clinical view after dental implant treatment in MRONJ patient using denosumab. B. Panoramic radiography showed sequestrum line around of the dental implant at the right mandible molar area.

Onur Şahin et al: Risk factors of medication-related osteonecrosis of the jaw: a retrospective study in a Turkish subpopulation. J Korean Assoc Oral Maxillofac Surg 2019

In Group I, rates of use for different drugs that cause MRONJ were similar. Half of patients were using ibandronate $(52.2 \%)$ and the other half denosumab (47.8\%) together with zoledronate. In Group II, alendronate (72.4\%) was the most common drug causing MRONJ.

The reason that BPs cause osteonecrosis in the jaw bones rather than other bones is that BPs accumulate at high levels in alveolar bones with high regenerative capacity and in soft tissue adjacent to the bones. The inability to create an aseptic environment due to the large microflora of the oral cavity, the constant association with this microflora through the periodontal gap, and a high trauma incidence increase the risk of osteonecrosis in the jaw bones ${ }^{17}$. Marx et al. ${ }^{18}$ reported that $68.1 \%$ of MRONJ cases were mandibular and $27.7 \%$ were maxillary in their study. Similar results were reported by Badros et al. ${ }^{19}$. According to their study on multiple myeloma patients with BRONJ, 15 patients had mandibular, 2 had only maxillary and 5 had both mandibular and maxillary lesions. In our study, 14 cases were mandibular, 8 cases were maxillary and 1 case was in both jaws in Group I. In Group II, 16 cases were mandibular, 12 cases were maxillary and 1 case of MRONJ was in both jaws. MRONJ was found in the mandibles of 30 patients.
In the cohort study conducted by Vahtsevanos et al. ${ }^{20}$, they calculated the relative risk for osteonecrosis of the jaw (ONJ) development to be 18 times higher in patients who experienced dental extractions and patients who use dentures had an at least two-fold increased risk for ONJ development. Dental extraction alone is a predisposing factor for $52 \%$ to $61 \%$ of examples of MRONJ formation ${ }^{21}$. The incidence of osteonecrosis after tooth extraction in patients using lowconcentration BPs is reported to be $0.5 \%$; it is reported to be $1.6 \%$ to $14.8 \%$ in patients receiving high-concentration $\mathrm{BPs}^{22}$. In our study, the factor that caused the formation of MRONJ was usually tooth extraction, in accordance with previous study ${ }^{20-22}$. In Group I, 14 of patients had MRONJ after tooth extraction, 6 after receiving dental prosthetics and 2 had spontaneous MRONJ. In addition, we observed osteonecrosis of the right mandible after dental implant therapy due to use of zoledronate and denosumab.(Fig. 3) Of patients in Group II, 15 had MRONJ post tooth extraction, 6 because of prosthetics, and 8 had spontaneous MRONJ. In both groups, the etiologic factor that caused MRONJ was mostly tooth extraction.

Lesions were staged by AAOMS classification. The parameters examined were pain, suppuration, presence of exposed bone, infection, and jaw fractures. Bagan et al. ${ }^{23}$ and Fedele et al. ${ }^{24}$ reported the most frequently encountered symptoms as pain and necrotic bone. Bagan et al. ${ }^{23}$ found pain in $77 \%$ and necrotic bone in $69 \%$ of patients in their study. These two signs of MRONJ were the most common clinical symptoms in our study in accordance with previous studies. However, $70 \%$ of patients in our study had bone exposure and $63 \%$ had pain. In our study, exposed bone was more common than pain in both groups, but no significant difference was found between groups except for suppuration $(P=0.024)$.

Demographic and systemic factors and other drug use also affect MRONJ formation. Age and sex are risk factors for $\mathrm{MRONJ}^{25}$. The types of drugs that cause osteonecrosis are given more frequently to women due to their indicational status. In our study, the majority of patients with MRONJ were female: $78.3 \%$ in Group I and $93.1 \%$ in Group II. The mean age of patients in this present study is in accordance with the age of those in the literature ${ }^{23,25}$. We also observed correlations between patient age and lesion stage. The older the age, the higher the incidence of stage 2 and 3 disease. A significant difference was found in the relationship between age and stage. The incidence of stage 2 and 3 increased with age in patients with $\operatorname{MRONJ}\left(\chi^{2}=17.73, P<0.05\right)$.

Whether smoking is a risk factor for MRONJ is still be- 
ing discussed in the literature. Some studies find it to have an effect while others report that it is not a risk factor for MRONJ $^{17}$. In our study, 3 patients smoked 20 cigarettes per day for more than 10 years: 2 had stage 1 disease and one had stage 3 MRONJ lesions.

\section{Conclusion}

According to results of our study, high-concentration BP intake, age, and duration of drug intake increased disease severity. According to the AAOMS staging system, updated in 2014, clinical and radiological findings have great importance for diagnosing MRONJ. In studies to date, the pathogenesis of MRONJ was explained theoretically but the pathophysiology of this disease is not exactly understood, despite the long time since the first reported case of MRONJ in the literature. Future studies should aim to explain the pathogenesis of MRONJ and find effective treatment strategies in larger groups of patients. Before intake of any BP or antiresorptive drugs, patients with cancer or osteoporosis should have an extensive dental examination, especially by an oral and maxillofacial surgeon who is experienced in the prevention and treatment of MRONJ. Patients with a challenging dental situation should participate in dental care before starting these medications.

\section{ORCID}

Onur Şahin, https://orcid.org/0000-0001-7816-1443

Onur Odabaş1, https://orcid.org/0000-0001-7771-048X

Toghrul Aliyev, https://orcid.org/0000-0002-6312-476X

Birkan Tatar, https://orcid.org/0000-0002-8917-575X

\section{Authors' Contributions}

O.Ş., T.A., and B.T. participated in data collection and wrote the manuscript. O.Ş. and O.O. participated in the study design and coordination. O.O. and B.T. performed the statistical analysis. O.O. helped to draft the manuscript. All authors read and approved the final manuscript.

\section{Ethics Approval and Consent to Participate}

The study was approved by the Ethical Committee of İzmir Katip Çelebi University (approval No. 207).

\section{Consent for Publishing Photographs}

Written informed consent was obtained from the patients for publication of this article and accompanying images.

\section{Conflict of Interest}

No potential conflict of interest relevant to this article was reported.

\section{References}

1. Marx RE. Pamidronate (Aredia) and zoledronate (Zometa) induced avascular necrosis of the jaws: a growing epidemic. J Oral Maxillofac Surg 2003;61:1115-7.

2. Ruggiero SL, Dodson TB, Fantasia J, Goodday R, Aghaloo T, Mehrotra B, et al.; American Association of Oral and Maxillofacial Surgeons. American Association of Oral and Maxillofacial Surgeons position paper on medication-related osteonecrosis of the jaw--2014 update. J Oral Maxillofac Surg 2014;72:1938-56.

3. Rosen LS, Gordon D, Tchekmedyian NS, Yanagihara R, Hirsh $\mathrm{V}$, Krzakowski M, et al. Long-term efficacy and safety of zoledronic acid in the treatment of skeletal metastases in patients with nonsmall cell lung carcinoma and other solid tumors: a randomized, Phase III, double-blind, placebo-controlled trial. Cancer 2004;100:2613-21.

4. Benhamou CL. Effects of osteoporosis medications on bone quality. Joint Bone Spine 2007;74:39-47.

5. Woo SB, Hellstein JW, Kalmar JR. Narrative [corrected] review: bisphosphonates and osteonecrosis of the jaws. Ann Intern Med 2006;144:753-61.

6. Ruggiero S, Gralow J, Marx RE, Hoff AO, Schubert MM, Huryn $\mathrm{JM}$, et al. Practical guidelines for the prevention, diagnosis, and treatment of osteonecrosis of the jaw in patients with cancer. J Oncol Pract 2006;2:7-14.

7. Hokugo A, Christensen R, Chung EM, Sung EC, Felsenfeld AL, Sayre JW, et al. Increased prevalence of bisphosphonate-related osteonecrosis of the jaw with vitamin D deficiency in rats. J Bone Miner Res 2010;25:1337-49.

8. Ruggiero SL, Dodson TB, Assael LA, Landesberg R, Marx RE, Mehrotra B; Task Force on Bisphosphonate-Related Osteonecrosis of the Jaws, American Association of Oral and Maxillofacial Surgeons. American Association of Oral and Maxillofacial Surgeons position paper on bisphosphonate-related osteonecrosis of the jaw 2009 update. Aust Endod J 2009;35:119-30.

9. Campisi G, Di Fede O, Musciotto A, Lo Casto A, Lo Muzio L, Fulfaro F, et al. Bisphosphonate-related osteonecrosis of the jaw (BRONJ): run dental management designs and issues in diagnosis. Ann Oncol 2007;18 Suppl 6:vi168-72.

10. Kim TH, Seo WG, Koo CH, Lee JH. Evaluation of the predisposing factors and involved outcome of surgical treatment in bisphosphonate-related osteonecrosis of the jaw cases including bone biopsies. J Korean Assoc Oral Maxillofac Surg 2016;42:193-204.

11. Ruggiero SL, Fantasia J, Carlson E. Bisphosphonate-related osteonecrosis of the jaw: background and guidelines for diagnosis, staging and management. Oral Surg Oral Med Oral Pathol Oral Radiol Endod 2006; 102:433-41.

12. Zervas K, Verrou E, Teleioudis Z, Vahtsevanos K, Banti A, Mihou $\mathrm{D}$, et al. Incidence, risk factors and management of osteonecrosis of the jaw in patients with multiple myeloma: a single-centre experience in 303 patients. Br J Haematol 2006;134:620-3.

13. Pazianas M, Miller P, Blumentals WA, Bernal M, Kothawala P. A 
review of the literature on osteonecrosis of the jaw in patients with osteoporosis treated with oral bisphosphonates: prevalence, risk factors, and clinical characteristics. Clin Ther 2007;29:1548-58.

14. Lo JC, O'Ryan FS, Gordon NP, Yang J, Hui RL, Martin D, et al. Prevalence of osteonecrosis of the jaw in patients with oral bisphosphonate exposure. J Oral Maxillofac Surg 2010;68:243-53.

15. Berenson JR, Yellin O, Crowley J, Makary A, Gravenor DS, Yang $\mathrm{HH}$, et al. Prognostic factors and jaw and renal complications among multiple myeloma patients treated with zoledronic acid. Am J Hematol 2011;86:25-30.

16. Dimopoulos MA, Kastritis E, Anagnostopoulos A, Melakopoulos I, Gika D, Moulopoulos LA, et al. Osteonecrosis of the jaw in patients with multiple myeloma treated with bisphosphonates: evidence of increased risk after treatment with zoledronic acid. Haematologica 2006;91:968-71.

17. Ruggiero SL, Mehrotra B, Rosenberg TJ, Engroff SL. Osteonecrosis of the jaws associated with the use of bisphosphonates: a review of 63 cases. J Oral Maxillofac Surg 2004;62:527-34.

18. Marx RE, Sawatari Y, Fortin M, Broumand V. Bisphosphonateinduced exposed bone (osteonecrosis/osteopetrosis) of the jaws: risk factors, recognition, prevention, and treatment. J Oral Maxillofac Surg 2005;63:1567-75.

19. Badros A, Weikel D, Salama A, Goloubeva O, Schneider A, Rapoport A, et al. Osteonecrosis of the jaw in multiple myeloma patients: clinical features and risk factors. J Clin Oncol 2006;24:945-
52.

20. Vahtsevanos K, Kyrgidis A, Verrou E, Katodritou E, Triaridis S, Andreadis CG, et al. Longitudinal cohort study of risk factors in cancer patients of bisphosphonate-related osteonecrosis of the jaw. J Clin Oncol 2009;27:5356-62.

21. Henry DH, Costa L, Goldwasser F, Hirsh V, Hungria V, Prausova $\mathrm{J}$, et al. Randomized, double-blind study of denosumab versus zoledronic acid in the treatment of bone metastases in patients with advanced cancer (excluding breast and prostate cancer) or multiple myeloma. J Clin Oncol 2011;29:1125-32.

22. Yamazaki T, Yamori M, Ishizaki T, Asai K, Goto K, Takahashi K, et al. Increased incidence of osteonecrosis of the jaw after tooth extraction in patients treated with bisphosphonates: a cohort study. Int J Oral Maxillofac Surg 2012;41:1397-403.

23. Bagan JV, Jimenez Y, Murillo J, Hernandez S, Poveda R, Sanchis $\mathrm{JM}$, et al. Jaw osteonecrosis associated with bisphosphonates: multiple exposed areas and its relationship to teeth extractions. Study of 20 cases. Oral Oncol 2006;42:327-9.

24. Fedele S, Porter SR, D'Aiuto F, Aljohani S, Vescovi P, Manfredi M, et al. Nonexposed variant of bisphosphonate-associated osteonecrosis of the jaw: a case series. Am J Med 2010;123:1060-4.

25. Hoff AO, Toth BB, Altundag K, Johnson MM, Warneke CL, Hu $\mathrm{M}$, et al. Frequency and risk factors associated with osteonecrosis of the jaw in cancer patients treated with intravenous bisphosphonates. J Bone Miner Res 2008;23:826-36. 